\title{
OS EFEITOS DA AROMATERAPIA EM PACIENTES COM ALZHEIMER: UMA REVISÃO NARRATIVA DA LITERATURA
}

\author{
Jaqueline Stefanello Garlet ${ }^{1}$; Adriele Saccol Nunes'; Thalia Teixeira Brum \\ Mengue $^{3}$; Lucila de Bortoli da Silva ${ }^{1}$; Larissa Gasparini da Rocha ${ }^{2}$; Nadiesca \\ Taisa Filippin ${ }^{6}$; Sheila Spohr Nedel ${ }^{7}$.
}

\section{RESUMO}

Objetivo: Esse trabalho é uma revisão da literatura sobre os benefícios da aromaterapia em pacientes com doença de Alzheimer. Metodologia: Este estudo caracteriza-se como uma revisão narrativa da literatura, onde foi feita uma pesquisa sobre os efeitos da aromaterapia em pacientes com doença de Alzheimer, através de estudos retirados da base de dados PubMed. Os dados encontrados foram analisados e selecionados utilizando critérios de inclusão e exclusão. Resultado: A busca na base de dados PubMed resultou na coleta de 218 artigos, onde 4 responderam adequadamente os critérios de inclusão, 77 eram revisão de literatura e 148 estavam fora do tema proposto. Conclusão: Foram utilizados nas pesquisas os óleos essenciais Cedrela odorata, Salvia Sclarea e Melissa officinalis. Onde o Cedrela odorata e Melissa officinalis demonstraram melhora significativa de sintomas comportamentais e psicológicos e o sal de banho de Salvia Sclarea não evidenciou mudanças durante a intervenção.

Palavras-chave: Aromaterapia e Alzheimer

Eixo Temático: Atenção Integral e Promoção à Saúde.

\section{INTRODUÇÃO}

A doença de Alzheimer (DA) é uma patologia neurodegenerativa que acomete funções cerebrais como memória, linguagem, cálculo, comportamento e são

\footnotetext{
${ }^{1}$ Jaqueline Stefanello Garlet; ${ }^{2}$ Adriele Saccol Nunes e ${ }^{3}$ Thalia Teixeira Brum Mengue -

Acadêmicas do Curso de Fisioterapia da Universidade Franciscana - jaqueline.garlet@un.edu.br ; adriele.nunes@ufn.edu.br ; t.brum@ufn.edu.br

${ }^{4}$ Lucila de Borloti da Silva - Orientadora externa _ luciladendrus@gmail.com

${ }^{5}$ Larissa Gasparini da Rocha; ${ }^{6}$ Nadiesca Taisa Filippin e ${ }^{7}$ Sheila Spohr Nedel _

Orientadoras Docentes do Curso de Fisioterapia da Universidade Franciscana larissa.rocha@ufn.edu.br ; nadifilipin@ufn.edu.br Sheila.nedel@gmail.com;
} 
comprometidas de forma lentamente progressiva levando o paciente a uma dependência para executar suas atividades de vida diária. (Sereniki et al. 2008).

A patogenia da DA é esclarecida pela hipótese amilóide, segundo a qual a deposição e o acúmulo do $\beta$-peptídeo amilóide $(A \beta)$ são responsáveis pela neurodegeneração; assim, a redução das placas $A \beta$ deve produzir melhora clínica. (Scutere D, et al. 2017).

A aromaterapia é uma parte especializada da fitoterapia, onde são extraídos óleos essenciais de diversas partes de plantas aromáticas. Desde a década de 80 , houve um aumento no interesse e pesquisa sobre óleos essenciais, pois foi sendo comprovada a eficácia do mesmo em vários transtornos, como ansiedade e transtorno de humor.

Além disso, junto com os tratamentos psicológicos medicamentosos, a aromaterapia tem comprovado grande eficácia para o tratamento da agitação, da agressão, sintomas psicológicos e comportamentais específicos da doença de Alzheimer. (Scutere D, et al. 2017).

Considerandando a eficácia da aromaterapia em alguns transtornos, como o transtorno de ansiedade, o presente estudo, teve como objetivo uma revisão da literatura sobre os benefícios da aromaterapia em pacientes com doença de Alzheimer.

\section{METODOLOGIA}

Este estudo caracteriza-se como exploratório, do tipo revisão narrativa da literatura. Onde foi feita a busca pelos estudos em setembro de 2021, na base de dados PubMed, os descritores e palavras-chave utilizados foram: aromatherapy, Alzheimer, seguidos do operador booleano AND.

Foram incluídos nesta revisão de literatura artigos completos, que abordam sobre o uso de óleos essenciais e extratos de óleos como tratamento das doenças neurodegenerativas do sistema nervoso central, na doença de Alzheimer. Estudos de revisão bibliográfica, estudos modelo animal e outras formas de demência que não sejam alzheimer foram excluídos da amostra. Todas as etapas da análise de qualidade metodológica dos artigos foram realizadas por dois avaliadores 
UCAÇÃO, SAÚDE

ETECNOLOGIA

26 A 28 DE OUTUBRO DE 2021

independentes e cegos. Após a busca no banco de dados, os títulos e resumos foram avaliados e os estudos que incluíram os critérios de inclusão foram selecionados para leitura na íntegra.

\section{RESULTADOS E DISCUSSÕES}

A busca na base de dados PubMed resultou na coleta de 218 artigos, onde 3 responderam adequadamente os critérios de inclusão, 77 eram revisão de literatura $e$ 148 estavam fora do tema proposto. As publicações que compuseram a amostra do presente estudo foram publicadas entre os anos de 2003 a 2020 conforme mostra a Tabela 1.

Tabela 1- Publicações que compuseram o presente estudo

\begin{tabular}{|c|c|c|c|}
\hline $\begin{array}{c}\text { Autor - ano } \\
\text { de } \\
\text { publicação }\end{array}$ & $\begin{array}{c}\text { Modelo de } \\
\text { estudo }\end{array}$ & $\begin{array}{l}\text { Espécie } \\
\text { utilizada }\end{array}$ & Objetivo do estudo \\
\hline $\begin{array}{l}\text { Takahashi Y, } \\
\text { et al (2020) }\end{array}$ & $\begin{array}{l}\text { In Vivo } \\
\text { (Humano) }\end{array}$ & Cedrela odorata & $\begin{array}{l}\text { Nós examinamos se os } \\
\text { sintomas de demência } \\
\text { são melhorados pela } \\
\text { estimulação do nervo } \\
\text { olfatório em pacientes } \\
\text { com } \\
\text { demência do tipo } \\
\text { Alzheimer. }\end{array}$ \\
\hline $\begin{array}{l}\text { Kouzuki M, et } \\
\text { al (2019) }\end{array}$ & $\begin{array}{l}\text { In vivo } \\
\text { (Humano) }\end{array}$ & Salvia Sclarea & $\begin{array}{l}\text { Avaliar os efeitos do óleo } \\
\text { aromático como um sal de } \\
\text { banho na função } \\
\text { cognitiva, função olfativa e } \\
\text { qualidade do sono. }\end{array}$ \\
\hline
\end{tabular}




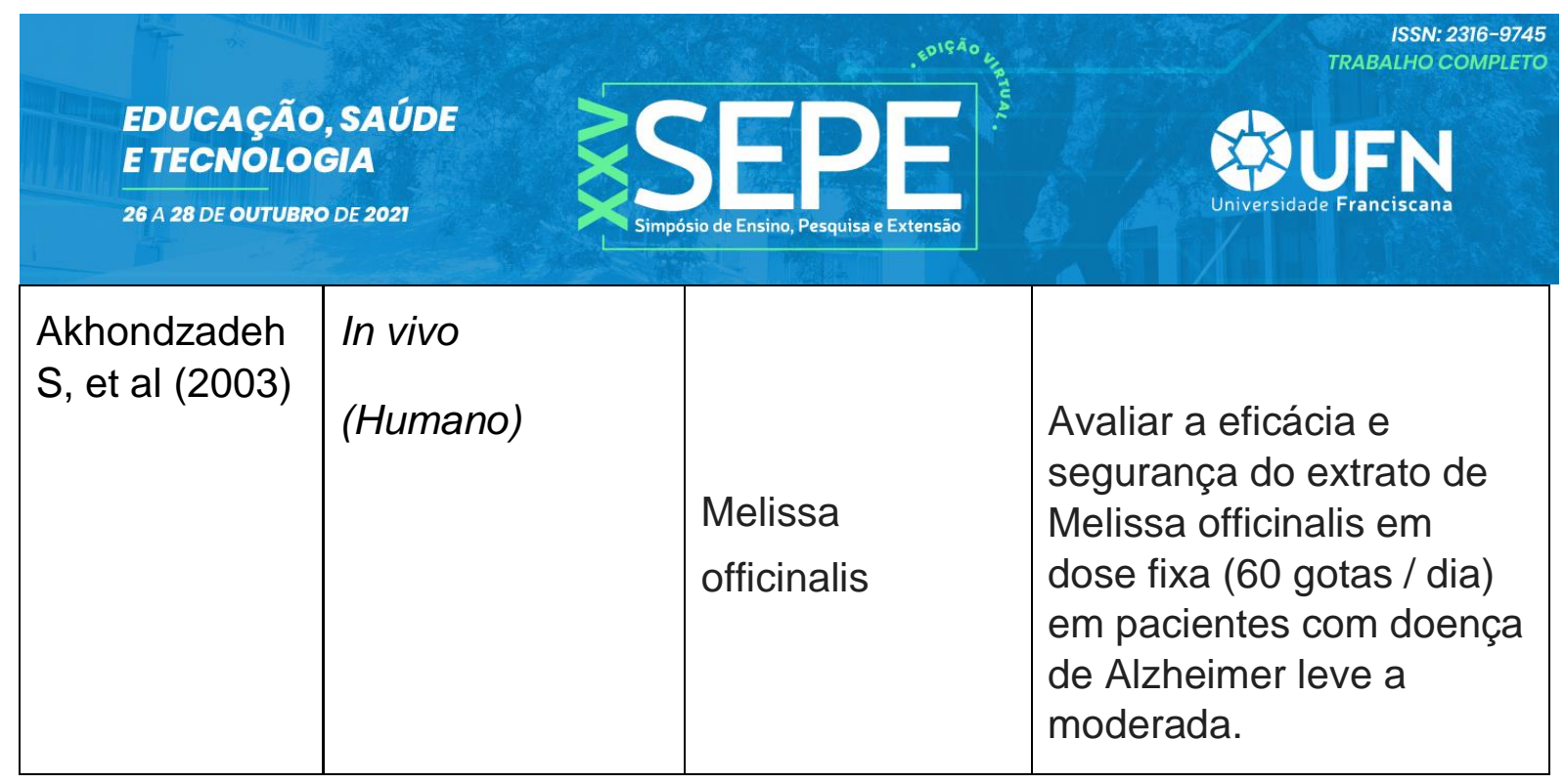

Fonte: Elaborada pelos autores

Conforme o estudo de Takahashi et al, (2020) examinaram os sintomas de demência e se são melhorados pela estimulação do nervo olfatório em pacientes com demência do tipo Alzheimer. Primeiro eles fizeram um teste de capacidade de identificação olfativa do tipo bastão nos pacientes, para selecionar pacientes sem disfunções olfativas. Os pacientes foram aleatoriamente designados para os grupos de intervenção $(n=19)$ onde expuseram eles a um etanol desinfetante com extrato de cedro e o controle $(n=17)$ ao etanol sem os extratos aromáticos adicionados com uma intervenção de 8 semanas, as funções cognitivas e comportamentais foram avaliadas antes e após os tratamentos. Houve uma melhora significativa no grupo de intervenção em comparação com o grupo de controle em 4 e 8 semanas. A exposição à fragrância de cedro melhorou os sintomas comportamentais e psicológicos da demência (BPSD) na demência do tipo Alzheimer. Além de sua eficácia, o procedimento é simples e minimamente invasivo e seria um tratamento não farmacêutico valioso. A exposição à fragrância de cedro melhorou os sintomas comportamentais e psicológicos da demência (BPSD) na demência do tipo Alzheimer e pode reduzir a carga de cuidados de enfermagem. Além de sua eficácia. A exposição à fragrância de cedro melhorou os sintomas comportamentais e psicológicos da demência (BPSD) na demência do tipo Alzheimer.

Segundo o Kouzuki M, et al (2019) realizou um ensaio clínico randomizado. Onde 49 pacientes assinaram o termo de consentimento e 35 foram finalmente analisados (doença de Alzheimer: 10, comprometimento cognitivo leve: 25). Os pacientes foram designados para usar sal de banho aromático a $0,1 \%, 0,5 \%$ ou $1 \%$. Durante o banho diário, o sal de banho foi adicionado à água do banho, e os indivíduos 
permaneceram no banheiro por $\geq 10$ min. A intervenção durou 24 semanas, e o tempo de observação foi de 4 semanas antes e depois de usar o sal de banho aromático. Para avaliar foi usado o Touch Panel-type Dementia Assessment Scale (TDAS), o Odor Stick Identification Test para o japonês (OSIT-J) e a versão japonesa do Índice de Qualidade do Sono de Pittsburgh (PSQI-J) cinco vezes durante os períodos de observação antes e depois e após a intervenção de 12 semanas. Foi percebido que não houve mudanças significativas nos escores TDAS, OSIT-J e PSQI-J antes e depois da intervenção em todos os grupos.

Akhondzadeh S, et al (2003) avaliaram a eficácia e segurança de uma dose fixa de Melissa officinalis durante um período de quatro meses em pacientes com doença de Alzheimer leve a moderada, usando um desenho de ensaio clínico duplocego, randomizado e controlado por placebo. Fizeram parte do estudo 42 pacientes, (18 mulheres, 24 homens) com doença de Alzheimer leve a moderada com idade entre 65 e 80 anos, com pontuação $\geq 12$ na subescala cognitiva da escala de avaliação da doença de Alzheimer (ADAS-cog) e $\leq 2$ em a classificação clínica de demência (CDR).

Os pacientes foram randomizados para receber extrato de Melissa officinalis 60 gotas / dia ou placebo 60 gotas / dia e deveriam manter contato regular com um cuidador responsável. O estudo mostrou que pacientes com doença de Alzheimer leve a moderada recebendo extrato de Melissa officinalis experimentaram benefícios significativos na cognição após as 16 semanas de tratamento, sendo as principais medidas de eficácia o ADAS-cog e o CDR-SB. O presente estudo não relata diferenças significativas nos dois grupos em termos de efeitos colaterais observados, exceto agitação, que foi mais comum no grupo placebo. O extrato de Melissa officinalis é valioso no tratamento da doença de Alzheimer leve a moderada e tendo um efeito positivo na agitação desses pacientes.

\section{CONCLUSÃO}

Pode-se concluir com essa revisão que existem evidências científicas acerca da utilização do extrato de Cedrela odorata, óleo aromático Salvia Sclarea e do óleo essencial de Melissa officinalis na melhora de sintomas desses pacientes. $O$ extrato de Cedrela odorata melhorou os sintomas comportamentais e psicológicos da 
demência.A Salvia Sclarea utilizada como sal de banho não evidenciou mudanças significativas nos escores desses pacientes nem antes e nem após a intervenção.

E o óleo essencial de Melissa officinalis demonstrou efeitos benéficos na cognição e agitação em pacientes com doença de Alzheimer de leve a moderada. Dessa forma acredita-se que a aromaterapia apresenta uma atividade promissora no tratamento de déficits cognitivos e comportamentais em pacientes com a doença de Alzheimer.

\section{REFERÊNCIAS}

AKHONDZADEH, S. et al. Extrato de Melissa officinalis no tratamento de pacientes com doença de Alzheimer leve a moderada: um ensaio duplo-cego, randomizado e controlado por placebo. Jornal de neurologia, neurocirurgia e psiquiatria. $v 74, n$ 7, p 863-6, 2003.

KOUZUKI, M. et al. Exame da influência da fragrância de cedro na função cognitiva e nos sintomas comportamentais e psicológicos da demência na demência do tipo Alzheimer. Neuropsicofarmacologia. v 41, n 1, p 10-15, 2019.

SERENIKI, A.; VITAL, M.A.B.F. A doença de Alzheimer: aspectos fisiopatológicos e farmacológicos. Revista de psiquiatria do Rio Grande do Sul, v. 30 n 1, 2008.

SCUTERE, D. et al. Aromaterapia e Plantas Aromáticas para o Tratamento dos Sintomas Comportamentais e Psicológicos da Demência em Pacientes com Doença de Alzheimer: Evidências Clínicas e Possíveis Mecanismos Evid Based Complement Alternat Med. 2017, 30 de março.

TAKAHASHI, Y. et al. Avaliação do efeito do óleo do aroma como um sal de banho na função cognitiva. Psicogeriatria. v 20, n 2, p 163-171, 2020. 